\title{
Gobernabilidad en las áreas protegidas y participación ciudadana
}

\author{
Carmen Sanz López \\ Adolfo José Torres Rodríguez \\ Universidad de Granada
}

\section{Resumen}

El V Congreso Mundial de Parques, organizado por la UICN en 2003, significa el inicio de una nueva etapa en las estrategias de conservación de la naturaleza y plantea la necesidad de aplicar un enfoque innovador para gestionar las áreas protegidas. En el artículo, se aportan una serie de reflexiones ordenadas sobre algunas de las causas que obstaculizan la gobernabilidad de las mismas. La propuesta que se articula se enmarca en los planteamientos de S. O. Funtowicz y J. R. Ravetz sobre «La ciencia posnormal. Ciencia con la gente» y persigue contribuir a la gestión de los espacios naturales protegidos y su evaluación de manera eficaz. Sobre todo, cuando nuestro interés se centra en aquellos aspectos relacionados con la participación de las comunidades locales.

Palabras clave: sociología ambiental, conservación de la naturaleza, ciencia posnormal, evaluación.

\section{Abstract. Governability in Protected Areas and Citizens Participation}

The $5^{\text {th }}$ World Parks Conference organized by the IUCN in 2003 means the beginning of a new era for nature conservation strategies and brings out the need to establish an innovative approach to manage the protected areas. This paper contributes a series of ordered reflections on some of the causes that hinder the management of such areas. This proposal is part of the approaches presented by S. O. Funtowicz and J.R. Ravetz on «Post-normal science. Science and people» and aims to contribute to the management and assessment of the protected areas in a useful way. Especially, taking into account that our interests are focussed on those aspects connected with the involvement of local communities.

Key words: environmental sociology, nature conservation, post-normal science, assessment.

\section{Sumario}

1. Introducción 4. La práctica de la participación

2. El nuevo marco en las estrategias de conservación de la naturaleza a partir de Durban

3. Gobernabilidad en las áreas protegidas ciudadana en las áreas protegidas 5. Reflexiones para un nuevo marco de gobernabilidad en las áreas protegidas Bibliografía 


\section{Introducción}

En el análisis de las áreas protegidas (AP), entendemos, de manera general, que la expresión "conservación de la naturaleza» resume en un solo concepto un amplio y diverso conjunto de actividades y medidas encaminadas al mantenimiento de los procesos ecológicos esenciales, la preservación de la diversidad genética y la utilización ordenada de los recursos naturales y culturales, así como su restauración y mejora (Europarc-España, 2002: 57). Este planteamiento asume que la conservación de espacios naturales implica protección, esto es, favorecer y/o defender los ecosistemas naturales para mantenerlos. Constatamos que la gran mayoría de espacios naturales que despiertan hoy interés para su conservación, o que lo hicieron en el pasado, han sido transformados y conservados por sus pobladores a través de sus hábitos de relación con el medio y su adaptación a él. Se puede afirmar, así, que las áreas protegidas son resultado de las actividades humanas en su interior. De otro lado, la actual sociología del medio ambiente ha incorporado como nuevo paradigma aquél que considera las condiciones de los sistemas medioambientales en los que se desenvuelven los sistemas sociales. De esta forma, las áreas protegidas han de enmarcarse en una noción dinámica del medio ambiente, en tanto éste no es algo externo al hombre ni a las sociedades. Este pensamiento sociológico parte del concepto de interacciones medioambientales o relaciones socioambientales como constructo teórico que asume la transición desde ecosistemas naturales hasta sistemas transformados antropológicamente, y viene a significar la transferencia de ciertas funciones físicas de conservación y realimentación desde el ecosistema natural hasta el sistema social (Woodgate y Redclift, 1998).

Todo espacio natural se corresponde de forma indudable con un ámbito geográfico absolutamente determinado. La novedad que aporta el concepto de área protegida y/o espacio natural protegido (AP o ENP) respecto a los planteamientos geográficos, será que la consideración definitiva viene sostenida por la existencia de una precisa consideración jurídica a la que es posible sumar nuevas consideraciones al concepto; sea ecológica, sociológica, económica, política, etc. Tanto a nivel internacional como estatal, existen diferentes tipologías o categorías de áreas protegidas, lo que origina una pluralidad de denominaciones y de instrumentos de planificación. La Unión Internacional para la Conservación de la Naturaleza (UICN) estableció, en el IV Congreso Mundial de Parques de 1992, celebrado en Caracas (Venezuela), seis figuras de protección. A saber:

- Reserva natural integral: las características que han de cumplir los espacios requieren no estar habitados ni usados de forma significativa y ser un área de gran valor. Los fines principales para su conservación son científicos y para la protección de la fauna silvestre.

- Parque nacional: se trata de un área manejada principalmente para la conservación de ecosistemas y con fines de recreación.

- Monumento natural: su vocación es de sitios significativos para la conservación de elementos específicos. Se trata de un área protegida manejada 
principalmente para la conservación de características naturales específicas.

- Área de manejo de hábitats o especies: manejada principalmente para la conservación con intervención a nivel de gestión.

- Paisaje terrestre y/o marítimo protegido: se trata de zonas extensas y manejadas principalmente para la conservación de paisajes y con fines recreativos.

- Área protegida con recursos manejados: se trata de un área protegida para la utilización sostenible de los ecosistemas naturales.

En el caso de España, el artículo 12 de la Ley 4/89, de 27 de marzo, de conservación de los espacios naturales y de la flora y fauna silvestre, diferencia cuatro categorías:

- Parques nacionales: son áreas naturales poco transformadas por la explotación u ocupación humana, que, en razón de la belleza de sus paisajes, la representatividad de sus ecosistemas o la singularidad de su flora, de su fauna o de sus formaciones geomorfológicas, poseen unos valores ecológicos, estéticos, educativos y científicos, cuya conservación merece una atención preferente.

- Reservas naturales: su creación tiene como finalidad la protección de ecosistemas, comunidades o elementos biológicos que, por su rareza, fragilidad, importancia o singularidad merecen una valoración especial.

- Monumentos naturales: se trata de espacios o elementos de la naturaleza constituidos básicamente por formaciones de notoria singularidad, rareza o belleza, que merecen ser objeto de una protección especial. Se consideran también formaciones geológicas, yacimientos paleontológicos y demás elementos de la gea que reúnan un interés especial por la singularidad o importancia de sus valores científicos, culturales o paisajísticos.

- Paisajes protegidos: lugares concretos del medio natural que, por sus valores estéticos y culturales, sean merecedores de una protección especial.

La declaración de un espacio natural como área protegida conlleva el reconocimiento implícito de «bien» o «recurso escaso» sobre la totalidad del espacio natural o hacia alguno de los elementos que lo integran. Supone una puesta en valor en la que subyace el no-reconocimiento de valor intrínseco a la naturaleza. A este respecto, J. Domenech (1999) plantea que, a menudo, las AP son islas de protección (por lo general, pequeñas) dentro de un mar de explotación, por lo que se protegen especies y comunidades pero no procesos. Para este catedrático de ecología, cualquier política de protección de espacios naturales debe tener en cuenta dos principios fundamentales: a) las áreas protegidas no pueden consistir en islas de protección, pequeñas y solitarias, en medio del mar de explotación, abandono o degradación del resto del territorio; y b) todo el territorio es merecedor de protección, porque la biosfera no hace distingos y se extiende por todo el territorio. Lo que debe protegerse 
no son las cosas (determinadas especies o comunidades), sino los procesos, y no es posible encasillar tales procesos dentro de espacios limitados arbitrariamente.

Los modelos de conservación de la naturaleza, al considerar a ésta un recurso - mediante la atribución de valor-, la convierten en mercancía —objeto de mercado-, lo que genera una amplia gama de intereses que desencadena abundante conflictividad entre los diversos actores tendentes al establecimiento de nuevos equilibrios de poder. La protección que la conservación implica no puede ser sinónimo de abandono productivo o bloqueo de actividades. El abandono productivo de un espacio natural para facilitar su conservación conducirá ineludiblemente a su transformación e incluso a su degradación ecológica. Sólo el mantenimiento de su función productiva —o de la ausencia de ésta-, no sólo económica sino sobre todo ecológica y social, puede facilitar la conservación, pero entendida ahora como preservación (poner a cubierto anticipadamente de algún daño o peligro de riesgo). Cuando estos espacios dejan de responder a la función (usos/no-usos) que los ha generado y pasan a cumplir una función para la que no han sido diseñados, es cuando se transforman en espacios frágiles necesitados de protección. Otra cuestión será las consecuencias y los costes sociales en la configuración de las estructuras socioeconómicas ante la nueva situación generada. Y es que, como indica Artemio Baigorri: «[...] Cada modelo de producción, cada sistema productivo, precisa de una naturaleza funcionalmente adaptada a sus necesidades [...] En suma, tratar de mantener las actividades [...] es siempre la mejor defensa frente a las agresiones exteriores al ecosistema a proteger [...] Hacer otro tipo de consideraciones es puro romanticismo, inservible para el análisis social» (Baigorri, 1998: 104-107).

\section{El nuevo marco en las estrategias de conservación de la naturaleza a partir de Durban}

El V Congreso Mundial de Parques, «Beneficios más allá de las fronteras», organizado por la UICN en la ciudad sudafricana de Durban, en septiembre de 2003, abre una nueva etapa en las estrategias de conservación de la naturaleza. Los principios que deben regir el periodo que se inicia con el nuevo siglo plantean la necesidad de aplicar un enfoque innovador para gestionar las áreas protegidas. El enfoque estratégico adoptado persigue la eficacia, tanto en el desarrollo de lineamientos técnicos, como en el establecimiento de políticas de gestión de las AP. Los resultados del Congreso se recogen en los documentos obtenidos como productos primarios del mismo que habrán de ser sometidos a aprobación por el Congreso Mundial de la Naturaleza ${ }^{1}$, al ser la única asamblea de miembros de la UICN revestida de un mandato oficial. En dichos documentos (Acuerdo de Durban, Plan de Acción y Recomendaciones),

1. Congreso a celebrar en Bangkok (Tailandia), del 17 al 25 de noviembre de 2004, bajo el lema "Gente y Naturaleza. Una sola Tierra». 
se hace patente el nuevo paradigma: insertar las AP en programas más amplios de conservación y desarrollo, con el objetivo de forjar sinergias entre la conservación, el mantenimiento de los sistemas que sustentan la vida y la promoción de un desarrollo sostenible. Se reconoce a las áreas protegidas como espacios donde convergen los intereses naturales, culturales y espirituales en tanto lugares de biodiversidad, proveedores de bienes y servicios de los ecosistemas, fuente de recursos al desarrollo y zonas donde se transmiten culturas y sistemas de valores.

Destacamos, a continuación, algunas de las principales preocupaciones recogidas por los participantes en el Congreso (pertenecientes a diversos organismos, asociaciones y comunidades locales de 154 países):

a) El número de áreas naturales y silvestres situadas fuera de las AP se ha reducido a la mitad en las dos últimas décadas.

b) En muchas de las áreas, la protección existe más en el papel que en la práctica, en especial en los países en desarrollo y en el medio marino.

c) Muchos de los costes de las AP se asumen a nivel local —en particular, por parte de las comunidades locales más pobres_-, mientras que sus beneficios se reciben a nivel global y/o mundial y aún no se valoran suficientemente.

d) Existe un déficit de financiación de las áreas protegidas.

Entre las soluciones propuestas, sobresalen aquéllas que incorporan la resiliencia como criterio de selección, diseño y gestión de los sistemas de AP para garantizar su supervivencia; reconocen la relación indisociable entre las personas y las áreas protegidas, promoviendo la participación de las comunidades locales en la creación, declaración y gestión de las mismas, y establecen estrategias innovadoras y diversificadas de generación de ingresos para asegurar la entrada de recursos financieros que permitan recompensar por el suministro de bienes y servicios de ecosistemas. Todo ello como cambios a operar en los actuales sistemas de gestión de las AP, que se muestran ineficaces para hacer frente a las presiones y a los desafíos cada vez mayores con los que se encuentran, relacionados las más de las ocasiones con la pobreza, la globalización, la falta de seguridad y los cambios mundiales. El análisis de la situación actual pone de manifiesto la necesidad de integrar en las AP a todos los sectores, utilizando tanto conocimientos científicos como tradicionales y promoviendo la participación de las comunidades locales en su creación, declaración y gestión.

Si el documento Acuerdo de Durban supone una llamada a la acción a todas las partes interesadas en las áreas protegidas, El plan de acción es una sugerencia de actividades necesarias para potenciar los beneficios y mejorar la cobertura y el manejo de las AP. El listado que contiene no está cerrado, en tanto implica adoptar medidas o acciones a diferentes niveles, desde el internacional hasta el local, pasando por las autoridades responsables de cada AP. En todos los casos, las acciones requerirán ser desplegadas por medio de grupos de interesados múltiples como respuesta a los sistemas de gestión de AP que carecen de objetivos, valores y principios compartidos. De modo general, se 
trata de vincular las áreas protegidas a los programas mundiales de desarrollo sostenible como modo de aprovechar sus valores económicos y contribuir a la mejora de las condiciones de vida. De manera particular, corresponde a las autoridades responsables de un AP desarrollar estrategias y actividades encaminadas a la generación de ingresos a nivel local, promoviendo el aprovechamiento sostenible de los recursos y dando poder de decisión a las comunidades locales, mediante una participación activa en la protección de la diversidad biológica, para lograr su coexistencia con el desarrollo humano. Otras actividades sugeridas en el Plan de Acción contemplan que actualmente las estructuras, los conocimientos y las aptitudes requeridas para administrar las AP son más especializadas y amplias que en el pasado, por la importancia creciente de los factores sociales y culturales, y asumen que se ha de reconocer el valor de los conocimientos autóctonos y tradicionales y aprovecharlos eficazmente. Por ello, recomiendan proceder a la investigación científica y técnica para aumentar la capacidad efectiva de manejo, conociendo lo suficiente las tendencias de los indicadores ecológicos, sociales, culturales y económicos, y adoptar decisiones fundamentadas en la gestión de las áreas protegidas desde un enfoque integrador que posibilite un desarrollo local compatible con el manejo eficaz de las mismas.

\section{Gobernabilidad en las áreas protegidas}

Si el siglo pasado desarrolló y consolidó la declaración de áreas protegidas en el mundo, el nuevo siglo se inicia estableciendo como prioridad la gobernabilidad de las mismas. Los resultados del Congreso de Durban, a la par que recalcan la importancia que las AP revisten para el desarrollo sostenible, por la prestación de servicios ecológicos y por las oportunidades de sustento que representan, hacen notar el impacto negativo que dichas áreas pueden generar en las comunidades locales, si no se tienen en cuenta sus derechos y si éstas no participan plenamente en la elaboración y en la adopción de decisiones que les afectan. En este sentido, para contar con un sistema mundial de áreas protegidas debidamente gestionado, asumen la urgencia de medidas que permitan la participación activa en el proceso de la amplia variedad de sectores, comunidades e intereses presentes en cada caso. Una condición fundamental para lograr la responsabilización de los interesados directos será la creación de un ambiente de confianza y diálogo entre todas las partes. Dicho clima sólo se conseguirá mediante una buena gobernanza en las áreas protegidas. De acuerdo con esto, la gobernanza ocupa un lugar central para la conservación de las áreas protegidas. Se basa en una serie de principios subyacentes que representan lo que se podría describir como valores humanos fundamentales. Éstos incluyen el concepto de integración, oportunidades equitativas para contribuir al proceso de decisión, y una participación significativa de todas las partes afectadas por las áreas protegidas o que se beneficien con ellas. Comprende, asimismo, valores institucionales de transparencia, capacidad de dirección, eficiencia y rendición de cuentas. Los cinco principios de una «buena» gobernanza 
(legitimidad y voz, eficiencia, rendición de cuentas, equidad y dirección) permiten realizar el análisis comparativo de los diversos modelos existentes como proceso de adaptación de los mismos al contexto pertinente del AP concreta, y se convierten en instrumentos de evaluación participativa mediante ejercicios, como pueden ser los procesos participativos en la toma de decisiones, los procedimientos de resolución de conflictos y los mecanismos de control y evaluación. Las políticas apropiadas de gobernanza consistirán, entre otras cosas, en habilitar a las comunidades locales para participar en la ordenación de las AP, en asegurar una distribución equitativa de costos y beneficios relacionados con ellas, en reconocer la diversidad de enfoques relativos a la gobernanza y en conseguir una mayor disponibilidad de recursos para no hacer sobrellevar toda la carga a las comunidades locales y los beneficios a nivel mundial o nacional. El equilibrio entre costos y beneficios no siempre es de fácil definición en las AP, pues pocas veces se reconocen cabalmente las ventajas aportadas por ellas en forma de bienes y servicios ambientales (agua salubre y tierras de pastoreo, reservorio de poblaciones sostenibles de taxones de aguas costeras y de alta mar —incluyendo especies de importancia comercial—, amortiguación de contaminantes terrestres y aéreos, etc.).

Las AP son percibidas como la realización de una vocación ética. Expresan los deseos de la humanidad en pro de la preservación de la vida en el planeta. Por ello, son objeto de veneración. El grado en que las AP cumplen sus objetivos de conservación y alcanzan las metas sociales, económicas y ambientales está estrechamente relacionado con la calidad de su gobernanza. En la práctica, las AP deben administrarse de acuerdo con el enfoque por ecosistemas (estrategia de gestión integrada de las tierras, el agua y los recursos biológicos que promueve la conservación y la utilización sostenible de una manera equitativa), y por los principios de la UICN sobre las AP, que comprenden que la descentralización, la participación, la transparencia y la responsabilización deben tenerse en cuenta en todos los asuntos que afecten a los intereses recíprocos en las AP. En la actualidad, existe en el mundo una diversidad de tipos de gobernanza de áreas protegidas en función de las partes sobre las que debería recaer la responsabilidad de la misma sobre bases legítimas. El concepto de gobernanza abarca la interacción entre las estructuras, los procesos, las tradiciones y los sistemas de conocimientos que determinan la forma en que se ejerce el poder, la responsabilidad, se adoptan las decisiones y expresan su opinión los ciudadanos y otros interesados directos. Debe reflejar y abordar los factores sociales, ecológicos, culturales, históricos y económicos pertinentes y debe definirse teniendo en cuenta, para cada AP, las circunstancias, las tradiciones y los sistemas de conocimientos locales. Otro aspecto será el desarrollo y la aplicación de sistemas de evaluación de la eficacia de la gestión de las AP en tanto medio de aprendizaje basado en la experiencia. En este sentido, la vigilancia y las auditorías ambiental, socioeconómica e institucional forman un componente esencial de la gestión de las AP. Un programa de trabajo sobre la evaluación de la eficacia de la gestión de las AP debería comprender, al menos, la investigación y el desarrollo de indicadores, pautas y metodologías apropiados 
Cuadro 1. Fases de desarrollo de un área protegida como estructura de gestión.

\begin{tabular}{|c|c|c|c|c|}
\hline & Inicial & Desarrollo & Consolidación & Gestión activa \\
\hline Marco Legal & Declaración. & Sí. & Sí. & Sí. \\
\hline Planeamiento & $\begin{array}{l}\text { Sin planeamiento } \\
\text { o recién aprobado. }\end{array}$ & $\begin{array}{l}\text { En fase de } \\
\text { aprobación } \\
\text { y aplicación. }\end{array}$ & Sí. & Sí. \\
\hline Órgano gestor & $\begin{array}{l}\text { Sin órgano gestor } \\
\text { o no estructurado } \\
\text { o incompleto. }\end{array}$ & No estructurado. & $\begin{array}{l}\text { Sí, estructurado } \\
\text { y operativo. }\end{array}$ & $\begin{array}{l}\text { Sí, establecimiento } \\
\text { de procesos de } \\
\text { calidad. }\end{array}$ \\
\hline $\begin{array}{l}\text { Recursos } \\
\text { materiales }\end{array}$ & Insuficientes. & $\begin{array}{l}\text { Satisfechos } \\
\text { parcialmente. }\end{array}$ & Suficientes. & Adecuados. \\
\hline $\begin{array}{l}\text { Recursos } \\
\text { administrativos }\end{array}$ & Insuficientes. & Insuficientes. & Suficientes. & $\begin{array}{l}\text { Adecuados o } \\
\text { establecimiento } \\
\text { de procesos de } \\
\text { calidad y evaluación. }\end{array}$ \\
\hline $\begin{array}{l}\text { Recursos } \\
\text { económicos }\end{array}$ & $\begin{array}{l}\text { Insuficientes } \\
\text { y muy irregulares. }\end{array}$ & $\begin{array}{l}\text { Insuficientes. } \\
\text { Predominan } \\
\text { inversiones por } \\
\text { encima de gastos } \\
\text { de personal. }\end{array}$ & $\begin{array}{l}\text { Suficientes. } \\
\text { Incremento de } \\
\text { los costes de } \\
\text { personal con la } \\
\text { estabilización } \\
\text { de inversiones. }\end{array}$ & $\begin{array}{l}\text { Suficientes. } \\
\text { Programados con } \\
\text { tendencias regulares. }\end{array}$ \\
\hline
\end{tabular}

Fuente: Plan de Acción para los espacios naturales protegidos del Estado español.

para evaluar los aspectos relacionados con la gobernanza, y proporcionar un sistema general de criterios mínimos aplicable a nivel mundial que ofreciera un indicador general sistemático. De esta forma, los informes basados sobre sistemas de evaluación gozarían de credibilidad.

Para que un área protegida pueda ser objeto de seguimiento y evaluación en su gobernanza, es preciso que disponga, al menos, de los tres elementos siguientes: de un plan de gestión anual donde figuren los objetivos junto con los plazos para su consecución; de personal explícitamente dedicado a las actividades consignadas en el plan, y de un presupuesto. En este sentido, entendemos por gestión activa ${ }^{2}$ aquélla donde se identifican objetivos explícitos a los que se aplican medios materiales, humanos o de otro tipo claramente definidos y cuantificados. El concepto de gestión activa se agota en la dimensión «estructura de gestión» de las áreas protegidas y sus instrumentos mínimos (cuadro 1): marco legal, planeamiento, órgano gestor, recursos materiales, administrativos y económicos. Sin restar importancia a los avances que la gestión activa supone, habrá de reconocerse que el reto de futuro lo comporta el concepto

2. GÓMEZ-Limón, J. y otros (2000). De la declaración a la gestión activa. Los Espacios Naturales Protegidos del Estado Español en el umbral del siglo XXI, p. 37-41. 
de gobernanza. Una nueva etapa que marcará la evolución en la gestión de las $\mathrm{AP}$ al incorporar las necesidades y aspiraciones de las comunidades locales y otros interesados directos en las mismas mediante la participación de todos ellos en la toma de decisiones. Será necesaria, por tanto, una labor investigadora que permita la realización de estudios sobre los efectos que la gestión está teniendo con respecto a los múltiples y complejos objetivos que se reconocen a las áreas protegidas y sobre las carencias y lagunas que habrán de cubrirse en cuanto a su gestión y evaluación como prácticas de «buena gobernanza». El caso de la Unión Europea ilustra bien esta situación. El Informe de la Comisión, de enero de 2004, sobre la aplicación de la Directiva 92/43/CEE relativa a la protección de los hábitats naturales y de la fauna y flora silvestres - Directiva Hábitats - para el período 1994-2000, concluye que los estados miembros han progresado mucho respecto a la aplicación de las obligaciones de la directiva, sobre todo a nivel político y en el proceso de selección de espacios, aunque los resultados son muy decepcionantes en campos como el establecimiento de sistemas de vigilancia y control y la adopción de planes y objetivos de gestión. Así mismo, señala la conveniencia para el futuro de insistir más en la evaluación de la capacidad y los recursos humanos y técnicos utilizados en el objetivo de detener la pérdida de biodiversidad para el año 2010.

En el ámbito español, el Plan de Acción para los espacios naturales protegidos del Estado español (Europarc-España, 2002), en sus capítulos referidos a participación, investigación y evaluación, toma nota de las carencias a las que hemos de hacer frente en la gestión activa y sugiere algunas acciones con las que contamos para resolverlas y orientarnos hacia la gobernabilidad en los espacios naturales protegidos españoles. Comprobamos cómo la evaluación de la gestión en AP no está desarrollada en España y existe gran ambigüedad sobre la misma al diferenciar entre auditorías de regularidad y auditorías operativas o de rendimiento. Las primeras, consistentes en la revisión del gasto de las inversiones previstas o la evaluación de las actividades realizadas a lo largo de un año, son las que utilizan habitualmente la mayoría de administraciones públicas españolas. Las auditorías operativas se orientan a evaluar la eficacia y la eficiencia de la gestión inspiradas en criterios de equidad y son las que se realizan con menor frecuencia. En este segundo tipo de auditorías, suelen usarse indicadores de tipo ecológico, socioeconómico, de planificación y de gestión (el cuadro 2 muestra los indicadores más utilizados).

Entre las diferentes metodologías de evaluación de la gestión de AP, encontramos aquéllas que revisan el cumplimiento de los objetivos; los métodos económicos de coste/beneficio o coste/eficiencia; los métodos comprensivos que, además de valorar los efectos (esperados o no), consideran también los objetivos, los instrumentos y los medios utilizados, incluida la planificación del espacio natural, y los métodos basados en las percepciones de los usuarios, afectados o implicados que se utilizan a menudo para complementar a los métodos evaluadores del cumplimiento de objetivos. Con todo, se señala que faltan protocolos y metodologías rigurosas y fiables, por lo que se debe investigar y desarrollar un protocolo estándar de seguimiento que tenga en cuenta 
Cuadro 2. Indicadores más utilizados en auditorías operativas.

\begin{tabular}{lcc}
\hline Ecológicos & Sociales y económicos & Planificación y gestión \\
\hline - Diversidad (biótica & - Población (total, & - Normas legales y planes \\
y abiótica). & por sectores, pirámides, & de gestión (PRUG, \\
- Singularidad o rareza. & etc.). & PORN, Plan Especial \\
- Integridad & - Visitantes (número, & de Protección, etc.). \\
o naturalidad. & tipología, calidad, & - Planes de seguimiento. \\
- Fragilidad & satisfacción). & - Instrumentos \\
o vulnerabilidad. & - Conocimientos & evaluadores. \\
- Dimensiones. & y aceptación de & - Régimen de propiedad. \\
- Representatividad. & la población local. & - Gasto presupuestario. \\
- Conectividad. & Puestos de trabajo & - Dotación del equipo \\
& vinculados. & gestor. \\
& - Participación de & - Equipamientos. \\
& la población local. & - Niveles de protección. \\
& - Sectores productivos. & - Superficie. \\
& - Tasa de empleo. & \\
& - Rentas. & \\
& - Viviendas (primera & \\
& y segunda residencia).
\end{tabular}

Fuente: Plan de Acción para los espacios naturales protegidos del Estado español.

la tendencia de disminución de criterios técnicos y el aumento de la proyección social de los espacios protegidos (actualmente, menos del 7\% del seguimiento que se realiza, versa sobre aspectos socioeconómicos. Europarc-España, 2002, p. 131). De manera específica y en relación con la participación, destacamos dos de las acciones prioritarias señaladas en el Plan de Acción. La primera consistirá en realizar un «inventario de interesados» en sentido amplio; la segunda, en elaborar indicadores de participación de los interesados en la planificación y gestión.

\section{La práctica de la participación ciudadana en las áreas protegidas}

Si tratamos un área protegida determinada, tendremos que considerar todos los intereses presentes en la misma, evidenciando así que la gobernabilidad debe definirse para cada área protegida concreta. Se habrá de tener en cuenta, además de las peculiaridades naturales propias, la situación estructural que esté condicionando los comportamientos de los distintos grupos de actores. Esto es, considerar la forma concreta de la estructura y la organización social de las comunidades locales como variable para gestionar un AP. Hemos mostrado cómo las nuevas orientaciones de las estrategias de conservación de la naturaleza, al incorporar los factores sociales, económicos, políticos, culturales, 
etc., abandonan los criterios «naturalísticos» de carácter restrictivo. Actualmente, además del enfoque por ecosistemas, se asume que, para una buena gestión - activa - de las AP, se requiere de la evaluación de la misma; que para una buena gobernanza, se requiere de la participación de todos los sectores interesados, y que, en ambos casos, se requiere de investigaciones sobre procesos metodológicos e instrumental técnico que, incluyendo o al menos no olvidando los sistemas de conocimiento tradicionales, permitan determinar protocolos e indicadores dentro de un sistema de gestión tendente a la consecución de la «buena gobernanza» en la práctica.

Nuestra labor investigadora parte de estos planteamientos y toma al Parque Natural Sierra de Baza (PNSB) como unidad de análisis ${ }^{3}$. Este parque, declarado como tal por la ley andaluza $2 / 89$, de 18 de julio, es uno de los cuatro existentes en la provincia de Granada (España) y se encuentra situado en el extremo oriental de la misma. Sus 53.237 hectáreas protegidas pertenecen a los términos municipales de Baza, Caniles, Dólar, Gor y Valle del Zalabí (Charches). Por Decreto 122/1994, de 31 de mayo (BOJA de 21 de julio de 1994), se aprueban el Plan de Ordenación de los Recursos Naturales (PORN) y el Plan Rector de Uso y Gestión (PRUG). Engloba el conjunto orográfico constituido por las sierras de Baza y Gor, que forman parte de las Cordilleras Béticas. Se trata de un macizo montañoso que se eleva de los 900 a los 2.271 metros de altitud y que constituye una isla verde en un entorno semiárido: el altiplano granadino. El medio biofísico destaca por la relevancia territorial de las masas forestales y por su importancia hidrológica, al albergar numerosas cabeceras de cuencas. Se trata de una montaña mediterránea poblada desde antiguo, en la que, desde hace al menos siete mil años, ha tenido lugar una intensa relación entre la naturaleza y los seres humanos que la han poblado, fruto de la cual es la actual situación de la zona: un paisaje profundamente transformado pero que muestra una franca recuperación de sus valores naturales.

Los objetivos de la investigación se concretan en conocer los efectos de la gestión del Parque y las carencias y lagunas de gobernanza en relación con la participación social. El planteamiento metodológico diseñado diferencia dos momentos. En primer lugar, una aproximación descriptiva que permite presentar un perfil socioeconómico de los municipios del PNSB, similar a los indicadores socioeconómicos de las auditorías operativas, donde se analizan las características estructurales en cuanto a sistemas de poblamiento, características demográficas, estructura económica y sistemas productivos locales. En segundo lugar, una fase cualitativa que proporciona información sobre aspectos pertinentes de gobernanza, lo que permite un análisis sobre la realidad de la misma en el Parque y su extrapolación a nivel general. Los criterios de muestreo teórico aplicados en la selección de entrevistados (representación estratégica, heterogeneidad territorial y diversidad sectorial) permiten obtener el casi-

3. Torres RodrígueZ, A. J. (2003). Estudio sociológico de los espacios naturales protegidos: De la conservación a la sostenibilidad. Universidad de Granada. 
Cuadro 3. Casillero tipológico.

\begin{tabular}{ll}
\hline Ayuntamiento & $\begin{array}{l}\text { Grupos de gobierno. } \\
\text { Grupos de oposición. }\end{array}$ \\
\hline Propietarios en el parque & Agricultores y ganaderos. \\
& Propietarios residentes. \\
\hline Representantes de sectores empresariales & Comercio. \\
& Hostelería y restauración. \\
& Agrícola y ganadero. \\
& Recreativo-cultural. \\
& Construcción. \\
& Trabajos silvícolas. \\
\hline Asociaciones ciudadanas & Cazadores. \\
& Juveniles. \\
& Damnificados del PNSB. \\
& Culturales. \\
& Amas de casa. \\
& Protección civil. \\
& De vecinos. \\
\hline Técnicos de la Administración & Empleado en el Parque. \\
& Agente forestal. \\
& Gerente de la Agencia de Desarrollo Rural. \\
& Presidente de la Junta Rectora. \\
& Director conservador. \\
\hline
\end{tabular}

Fuente: Elaboración propia.

llero tipológico diseñado (cuadro 3) como forma de concretar el «inventario de interesados» en el Parque al que se hace referencia en el Plan de Acción para los espacios naturales protegidos del Estado español. En este sentido, la investigación permite apuntar líneas de actuación integradoras de todos los sectores interesados en el Parque tendentes a eliminar los obstáculos existentes actualmente, tanto en el diseño como en el funcionamiento, de los órganos de gestión y participación social del Parque Natural Sierra de Baza. A saber, el director conservador y la Junta Rectora, cuya regulación general está contenida en el título I del Plan Rector de Uso y Gestión.

El PNSB está dirigido por la Consejería de Medio Ambiente de la Junta de Andalucía. El director conservador es el máximo representante de la misma y el responsable del personal técnico y de los agentes que trabajan en el Parque. Asume la responsabilidad ejecutiva de la administración del Parque, del control de las actividades que en él se desarrollen y de la realización de las actuaciones ligadas a la conservación y al uso público. A su vez, se constituye como el nexo de conexión entre la Consejería de Medio Ambiente y la Junta Rectora, de la que es miembro nato.

La Junta Rectora (definida en el artículo 4 del PRUG - 1994- como «órgano colegiado de participación con la administración medioambiental y 
con funciones de coordinación de las administraciones públicas y colaboración ciudadana en la conservación del espacio protegido") queda integrada, a efectos administrativos, en la administración medioambiental y bajo supervisión de ésta, aunque goza de autonomía funcional y de organización en el ejercicio de las funciones consultivas y de participación que le son propias.

El artículo 12 del Reglamento de Régimen Interior (aprobado el 14 de abril de 1999 y publicado en BOJA de 8 de marzo de 2001) se refiere a las atribuciones de la Junta Rectora, de todas ellas destacamos: facilitar la participación de los habitantes del Parque Natural como garantía de preservación y desarrollo del espacio protegido; informar del programa anual de actuaciones, así como conocer y aprobar la memoria anual de actividades y resultados del Parque Natural, y ser oída previamente al nombramiento del director conservador del Parque. En cuanto a sus funciones y composición actual, el artículo 1 del citado reglamento contiene una nueva definición («órgano colegiado de control, vigilancia y participación ciudadana con la Consejería de Medio Ambiente, que velará por el cumplimiento de la normativa reguladora del Parque») que difiere de la establecida en el PRUG, aunque mantiene el carácter colegiado y de participación con la administración medioambiental. Es importante destacar este aspecto, en tanto que la participación ciudadana en la Junta Rectora del Parque Natural Sierra de Baza, y en general de todos los parques andaluces, ha sido concebida - tradicionalmentecomo "colaboración con la administración medioambiental». Es decir, como labor de apoyo a la acción político-administrativa medioambiental. Se suman, en la definición actual, las funciones de control y vigilancia de la labor administrativa (representada en el director conservador), pero sigue sin serle otorgada ni reconocida la función, diríamos que, legislativa en el funcionamiento del Parque Natural.

Apuntábamos anteriormente que los resultados obtenidos con nuestra investigación no se agotan en percepciones meramente descriptivas, sino que buscan configurar líneas concretas de gobernanza y gestión. En tal sentido, y en relación con el cargo de director conservador, identificamos en él al máximo responsable del Parque Natural, aunque su dedicación principal le ocupe en resolver tareas burocráticas. Se trata de un cargo con escasa capacidad operativa, sus actuaciones dependen de los proyectos que se aprueben por la Consejería de Medio Ambiente. Se podría apuntar, incluso, cierta dependencia política por la propia naturaleza del puesto, al tratarse de libre designación entre funcionarios de carrera. Entre los requisitos que debería reunir un director conservador «ideal», podemos señalar, por un lado, que el puesto tuviese un carácter más técnico y mayor especialización; por otro lado, habría de gozar de mayor autonomía y recursos para poder tomar decisiones en lo relativo a la realización de determinados proyectos en el Parque. Por último, debería contar con un equipo de personas que le asesorasen y le ayudasen en la difícil tarea de gestionar el Parque Natural. Como cuestiones relativas no al cargo sino a la persona, encontramos que debe mantener lazos con la zona, mostrar sensibilidad hacia las poblaciones locales y sentir un interés constante por el Parque. 
Las primeras consideraciones hacia la Junta Rectora se dirigen a destacar su carácter consultivo y la carencia de competencias ejecutivas. Este hecho la convertiría en un órgano poco operativo necesitado de un aldabonazo para hacerla más autónoma, tanto en la toma de decisiones como en la disponibilidad y manejo de fondos financieros. Se conseguiría con esto que sus miembros se considerasen participando de manera efectiva en la gestión del Parque. Otro aspecto que contribuye a la desazón existente con el funcionamiento de la Junta Rectora del PNSB tiene que ver con los temas o asuntos que ocupan las reuniones, muy especializados y/o de carácter administrativo. Es opinión compartida que no se plantean soluciones globales para el Parque ni se tratan temas que para las comunidades locales son importantes. Cabe destacar los relativos a puestos de trabajo en la sierra, la compra de fincas, la construcción y urbanización, la regulación y los problemas con los diferentes aprovechamientos y usos (cita 29: 6). Todo esto tendría como efecto desvirtuar la participación social (cita 3: 21), fin para el que la Junta Rectora fue diseñada. En este sentido, cobra suma importancia la composición de la misma. Utilizando criterios amplios de agrupación de sus miembros, se pueden establecer, grosso modo, tres grupos: representantes de las distintas consejerías de la Junta de Andalucía, miembros de los partidos políticos y representantes de diversos colectivos ciudadanos. Se critica la sobrerrepresentación percibida de la Administración, como medio de legitimación de la gestión que se realiza en el Parque. En la crítica subyace la imposibilidad — formal — de los representantes de las comunidades locales para imponer sus criterios de actuación al chocar con la «mayoría cualificada» de la Administración. El objetivo será conseguir una mayor presencia en la Junta Rectora de los colectivos de los municipios que forman el Parque, tanto en número de representantes como en el peso específico de sus planteamientos. Esta reivindicación sobre la participación local en la Junta Rectora encuentra como freno los recelos frente a los «localismos» (citas 7:29, $34: 12$ y $3: 24)$.

\section{Asuntos a tratar:}

\section{9:6 (99:123) [Junta Rectora]}

He estado dos veces y no voy más. Eso es el rollo más grande que hay en el mundo. Yo, las dos o tres veces que he estado, nunca he aprendido nada, ni he visto salir nada de provecho. Ahí nada más que hablan dos o tres de especies, claro, como uno no entiende, pero lo que no puede ser es que un tío se tire allí media hora hablando de la rama de pino o lo que sea y otro empiece a hablar de pocas cosas... Que no se habla de soluciones globales para el Parque. No sé, pocas cosas. Vamos, que yo no he visto nada claro en la Junta Rectora. Nada claro y nada de lo que se ha dicho yo he visto relucir. E1: EN TU OPINIÓN ¿QUÉ SE DEBERÍA TRATAR? P29: Temas más importantes. El trabajo en la sierra, dinero para el que tenga una finca y quiera darla al Parque... cosas más... pero no tanto... No hablar de temas tan pequeños para mi. Por ejemplo, será importante que haya un pino de una raza, importantísimo, pero vamos que eso no lo veo yo para estar media hora hablando del pino ese. Eso, se junta el científico ese que va y lo hable con el presidente o con el jefe del 
Parque, mira este pino hay que tener cuidado, hay que ponerle una valla... Pero eso no es razón para que nos junten allí a veinte personas de Graná, del quinto pino, para hablar de nada. Por lo menos, yo las dos veces que he estado... Te pegas $80 \mathrm{~km}$ para allá y otros para acá. Un día nevando... Si tratan otros temas, temas de trabajo, de aprovechamientos de leñas, aprovechamientos de agua, de aprovechamientos de caza...

\section{Participación social:}

\section{3:21 (234:239) [Junta Rectora]}

Yo le daría un funcionamiento más dinámico, algo en lo que se sintieran más partícipes los estamentos o las asociaciones que figuran, que tuvieran un poquito más de actividad, porque participar para nada es cargarse la participación, entonces habrá que darles un poco más de protagonismo.

Crítica y reivindicación:

\section{7:29 (229:233) [Junta Rectora]}

Deberían estar integrados más por personas de aquí y no políticos, y no lo digo por cosa personal. ¿Por qué un buen conocedor de Charches no puede estar en la Junta Rectora? Ya dice el refrán que más sabe el tonto en su casa que el listo en la ajena.

\section{4:12 (158:165) [Junta Rectora]}

La composición de la JR yo veo que hay cosas que no las entiendo. Todos los sectores de la sociedad de los municipios que incluye, que venga de la AMA estupendo, que de la Junta de Andalucía tienen que venir, magnífico, pero gente que sepa de qué va ahí que no sea política, que sepa de qué va esto. Y, lógicamente, yo no digo que se imponga lo que digan los representantes de los municipios, pero que se oiga, que se oiga muy bien, que son los que viven aquí.

\section{3:24 (261:265) [Administración responsable]}

E1: ¿¿SOBRE QUIÉN DEBERÍA RECAER LA MAYOR RESPONSABILIDAD EN LA GESTIÓN DEL PARQUE? P3: Creo que debe estar repartido, no me fío mucho de los municipios. Entiendo, en ese sentido, que una gestión provincial debe ser obligatoria. Lo que ocurre es que entiendo que la participación local debe ser como más activa, no tan simbólica. Debería fomentarse mucho más.

Las líneas de actuación mencionadas hasta ahora se refieren, de manera particular, al funcionamiento de los órganos de gestión y participación social del Parque Natural Sierra de Baza. Si nos referimos de manera general a la gobernanza de las AP, la investigación ha permitido delimitar un criterio común. Se trata de aquel planteamiento que establece que «quien tenga competencias para disponer del dinero, que tenga la responsabilidad». Será necesario reflexionar sobre esta idea en la nueva etapa que se inicia para la conservación de la naturaleza en las áreas protegidas a partir Durban. Según nuestro trabajo, el Parque Natural (en tanto área protegida) puede aportar inversiones tanto públicas 
como privadas a la zona. Para ello, sería necesario, por parte de la Administración que lo gobierna, primero, un compromiso presupuestario (elemento del que carece) y adoptar estrategias de descentralización política y desconcentración administrativa; segundo, fortalecer las estructuras de participación social para permitir la configuración de puntos comunes de encuentro entre todos los agentes sociales, económicos y políticos implicados en el mismo y conseguir un mayor control de los órganos gestores. La propuesta implica una flexibilización de las regulaciones coercitivas de conservación que permita lograr la coexistencia de la protección de la biodiversidad con el desarrollo humano, diferenciando entre los macroproyectos y las pequeñas actuaciones cotidianas. La solución pasa por asumir a las comunidades locales y conservar las aldeas (tanto las habitadas como las deshabitadas) en el marco de un nuevo sistema sociocultural de valores globalizado. La conservación de la naturaleza exige ahora un cuádruple compromiso: ecológico con la biodiversidad, político de participación social, económico para la financiación y la valoración ambiental, y social en pos de una distribución equitativa de los costes y los beneficios de las áreas protegidas. El resultado será una concreta forma de gobernanza con múltiples pequeñas acciones que, en conjunto, constituyen la solución total o general de gestión para cada AP, y son preludio de una ulterior forma de interacción o coevolución ${ }^{4}$ sostenible entre la sociedad y el medio biofísico que pueda «exportarse» al resto de espacios, sean o no protegidos.

\section{Reflexiones para un nuevo marco de gobernabilidad en las áreas protegidas}

$\mathrm{Si}$, al tratar la participación, es obvio que se estudia sobre un fenómeno político, será obvio también que no podamos olvidar el contexto comunitario y social que conforma la actitud y la práctica de la misma. Nuestro trabajo constata la complejidad del objeto de análisis por las múltiples perspectivas posibles y la pluralidad de factores intervinientes, así como el hecho de que la participación de las comunidades locales en la gobernabilidad de las áreas protegidas constituye una dimensión ineludible a tener en cuenta a la hora de gestionarlas. A continuación, presentamos, de manera sucinta, algunos de los obstáculos a la participación que nuestra investigación desvela. En tanto indicadores de participación la tarea que resta conlleva determinar los valores que cada uno de los factores considerados pueda adoptar. El análisis conjunto de todos ellos permite apuntar las causas, las dificultades y las trabas a una participación efectiva: el miedo o el temor a la participación, la falta de información sobre las ventajas de participar y las desiguales cotas de poder entre los distintos grupos de agentes:

4. El concepto de coevolución, de Richard Norgaard, que estaría en el punto de partida de lo que Woodgate y Redclift denominan relaciones socioambientales, enfatiza cómo las actividades de la gente «modifican los ecosistemas y cómo las respuestas de los ecosistemas proveen de un marco para la subsecuente acción individual y organización social» (Woodgate y Redclift, 1998, p. 27). 
a) Existe un grado de asociacionismo dispar entre los diferentes grupos de intereses implicados en el área protegida, de tal forma que algunos de los agentes sociales carecen de cauces formales para preparar y discutir los asuntos de las reuniones.

b) Se presentan problemas de distancia física (lugar) y tiempo (hora y duración) para la celebración de las reuniones que implican costes de tiempo y dinero (actividades que se dejan de realizar para poder asistir).

c) En algunos casos, los elegidos no son representativos del colectivo social al que representan.

d) El carácter ritual y falto de contenido de las reuniones. Los temas tratados se constituyen como campo de control (procedimientos y formalidades administrativas), lo que hace que los órganos de participación funcionen, en parte, como instancias administrativas de mera tramitación.

e) La duración total de las reuniones y el tiempo dedicado a cada tema particular muestran la desigual importancia o relevancia concedida a cada cuestión.

f) La frecuencia y duración de las intervenciones variará, distinguiendo entre los diversos representantes (se puede hablar de "convidados de piedra»).

g) Se produce un diferente grado de asistencia y absentismo a las reuniones según los distintos colectivos.

La participación implica asumir la parte de responsabilidad que corresponde a cada uno de los agentes sociales, no pudiendo atribuir al sujeto que padece el problema, es decir, que no participa o lo hace mínimamente, la falta de responsabilidad. Si bien la participación se ha convertido en un objetivo político en tanto requisito de gobernabilidad, en su puesta en práctica se produce una recontextualización por parte de los agentes sociales, dotándola de forma y contenido real alejado en ocasiones del sentido originario, de tal forma que la formación de la política de participación viene a encubrir la desigual distribución de las responsabilidades entre los agentes. La gobernabilidad de las AP requiere de instituciones participativas donde se produzca una participación efectiva. La determinación y consecución de los objetivos de la preservación de la naturaleza serán fruto de la directa imbricación entre los diferentes intereses particulares legítimos de los grupos que ponen algo en juego en la adopción de decisiones y en las diversas formas de conocimiento que le son propias. Se consigue, con esto, desterrar la incertidumbre institucional en la determinación de decisiones equitativas y la valoración de la participación como una forma de intrusismo, tanto activo como pasivo. El primer tipo, referido a la intromisión de colectivos sociales - de un supuesto estatus subordinado- en un terreno - preconcebido como propio— de expertos y gestores. En el segundo, los expertos y administradores confunden el saber cualificado — autoridad racional basada en el saber — con la participación —intereses particulares legítimos- Esto último concreta la gobernabilidad en las AP como proceso de toma de decisiones informado científicamente y participado socialmente de forma democrática bajo criterios de sostenibilidad. Si la metodolo- 
gía propuesta ha permitido su determinación mostrando obstáculos, evidenciando necesidades y registrando alternativas, todo apunta que «dar voz» a las necesidades y aspiraciones de las comunidades locales será útil también para proponer soluciones.

Los aspectos tratados constituyen el campo de nuestras actuales reflexiones sobre la gobernabilidad y la gestión de las áreas protegidas en aras de la preservación de la naturaleza. El contenido de las mismas se nutre de los argumentos que plantean Silvio Funtowicz y Jerome Ravetz, cuando, al tratar de la ciencia posnormal, formulan la necesidad de comunidades de pares extendidas como estructuras de toma de decisiones para la resolución de problemas complejos. Diseños participativos que se concretarían siguiendo, en el ámbito teórico, a John S. Dryzek, mediante prácticas de democracia discursiva como modelo más participativo de democracia y, en el terreno aplicado, a Joshua Cohen y Joel Rogers, con sus arenas deliberativas como mecanismos institucionales fruto de la imaginación organizativa.

En el caso de S. Funtowicz y J. Ravetz, la ciencia posnormal, asentada sobre una epistemología política, supone una directa imbricación entre conocimiento e intereses. Admite otras formas de pensamiento además del científico (ciencia pura, ciencia aplicada y consultoría profesional) en la resolución de problemas complejos como posibilidad de desarrollar elementos democráticos genuinos y efectivos si existe respeto mutuo entre las diversas perspectivas y formas de conocimiento que integrarían las comunidades de pares extendidas. La tarea consiste en elegir el tipo apropiado de estrategia científica para cada problema particular. Se trata de una ciencia cercana a una sociedad democrática que requiere de la participación extensiva y la tolerancia de la diversidad frente a la pretensión de racionalidad en la toma de decisiones públicas que debe parecer científica. La inclusión de cada vez más participantes en el proceso de reaseguro de la calidad de los inputs científicos exige, de un lado, establecer la legitimidad y la competencia de los participantes en las comunidades de pares extendidas; de otro lado, crear estructuras que conformen dichas comunidades. En el primer caso, la noción de diálogo posnormal - interactivo y creativo- implica ampliar el campo de los evaluadores de tal forma que «todos los que ponen algo en juego en las decisiones políticas, tienen su lugar en el diálogo", no sólo los expertos y administradores. Este criterio genera una situación de incertidumbre institucional que se disipa, para cada caso concreto, al considerar los agentes particulares (instituciones sociales y culturales, movimientos sociales, partidos políticos, etc.).

Se evidencia el carácter dinámico de la ciencia posnormal, al incluir los diversos aspectos del problema localizados en su zona y al utilizar el diálogo como medio para plantear, tanto los problemas como evaluar las soluciones. De forma similar al diálogo posnormal, John Dryzek presenta la interacción discursiva como "estilo conjunto y colectivo de resolver problemas complejos por parte de los individuos implicados». En su propuesta de democracia discursiva, señala que actualmente las instancias de poder buscan su propia legitimidad política mediante un modelo más participativo de democracia. Las 
decisiones políticas legítimas requieren ahora de conocimiento técnico, de apoyo de la autoridad constitucional y de participación informada de todas las partes implicadas. Se trata de procesos discursivos incipientes, al aproximarse al objetivo de un discurso libre, pero no constituyen esferas públicas autónomas, al estar asociadas con el Estado. Las prácticas discursivas, que requieren de muchos participantes y amplias discusiones, se sustentan en los ideales del discurso autónomo (ética comunicativa) en el cual el único poder legítimo es el del mejor argumento. La interacción comunicativa de los diseños discursivos (como estructuras participativas de resolución de problemas) constituye un medio descentralizado para proporcionar bienes públicos (soluciones generalizables a todas las partes implicadas), en la medida en que el discurso permite escrutar y penetrar las estructuras culturales e ideológicas. Las prácticas discursivas, si bien proporcionan legitimidad política a las instancias de poder, pueden conducir a su modificación por el debilitamiento de aquellos objetivos supuestamente no polémicos y de aparente neutralidad técnica (la definición y el funcionamiento de la Junta Rectora del Parque Natural Sierra de Baza son buenos ejemplos de este fenómeno). La propuesta que surge será la democratización en todos los niveles posibles, es decir, la democratización de la sociedad civil y del Estado. En este sentido, J. Cohen y J. Rogers plantean como estrategia la democracia asociativa, que implica una devolución de poder a la sociedad civil mediante una redefinición del papel del Estado (de solucionador de problemas a organizador de capacidades sociales para resolverlos).

En el discurrir de nuestra reflexión, confluyen en este punto tanto las dificultades de crear estructuras organizativas que conformen las comunidades de pares extendidas requeridas por la ciencia posnormal como las de articular diseños organizativos y de gobierno que permitan llevar a efecto las prácticas discursivas de las que trata Dryzek. La cuestión que estamos formulando en ambos casos será ¿cómo hacerlo? De nuevo, recurrimos a J. Cohen y a J. Rogers y a su propuesta de democracia asociativa, pero ahora como orden alternativo. En su estrategia de transferir determinadas responsabilidades del Estado a la sociedad civil, se necesita de asociaciones secundarias - arenas asociativaspara representar intereses más allá de las «arenas políticas formales» en tanto éstas no lo consiguen. Concretando, que si las instituciones existentes no lo proporcionan, habrá que construir nuevas arenas para la deliberación pública al margen de las arenas políticas convencionales que incluyan a negociadores no tradicionales. Esto es, diseñar un modelo institucional constructivo que parta del pluralismo y promueva el consenso político e incremente el aprendizaje social, simplificando la toma de decisiones y reduciendo los costes de supervisión. En ese modelo, el papel del Estado sería: apoyar la deliberación, establecer los requisitos para la inclusión de los participantes, asegurar la integridad del proceso y autorizar la estrategia concebida. A las nuevas instituciones, ellos las denominan "arenas deliberativas».

Aparentemente, habríamos resuelto el problema con la formación de arenas deliberativas; en nuestro caso, la creación de la Junta Rectora del Parque como órgano de participación social da legitimidad política (y científica) a las 
decisiones. El problema reside en que la participación no es efectiva. Primero, por el propio diseño de la política de participación, es decir, las «peculiaridades» en la definición, composición y funciones de la Junta Rectora y del director conservador del Parque. Segundo, por la recontextualización que efectúan los agentes sociales representados (incluida la Junta de Andalucía — director conservador y representantes de diversas consejerías-), producto de la carencia de una conciencia cívica en sus miembros que enraíce el ejercicio del poder político en prácticas de discusión pública entre ciudadanos iguales. Para finalizar, es interesante introducir, en la reflexión, la crítica que Iris Marion Young realiza a la democracia asociativa, basándose en la distinción entre asociación (institución formalmente organizada) y grupos sociales (colectivo de personas que se diferencia al menos de otro grupo por sus formas y prácticas culturales o por su estilo de vida) para proponer el principio de «representación especial de los grupos oprimidos» como modo de representación adecuada de todas las perspectivas de los grupos sociales en las arenas deliberativas. Entiende que los grupos sociales privilegiados tienden a estar bien representados en posiciones de poder e influencia en las instituciones, mientras que los grupos sociales oprimidos o de menores recursos económicos tienen escasa presencia o nula influencia en el establecimiento de la agenda política y/o en la elaboración de las políticas. Básicamente, este principio implica que el Estado promueva la autoorganización de los grupos sociales «oprimidos» y proporcione mayores recursos a las asociaciones que los representan para maximizar las oportunidades de tomar decisiones acertadas y equitativas, al permitir la expresión de necesidades e intereses diversos y al promover una orientación hacia un bien público que va más allá de la expresión de intereses particulares, al frenar la expresión, por parte de los grupos dominantes, de sus propios intereses como un bien general. De esta forma, estaríamos eliminando muchos de los obstáculos a la participación social en la gobernabilidad de las áreas protegidas que nuestra investigación ha puesto de manifiesto y reduciendo las causas que dificultan una participación efectiva: el miedo a la participación, la falta de información sobre las ventajas de participar y las desiguales cotas de poder entre los distintos grupos de agentes (de manera particular, la escasa influencia de las comunidades locales de las áreas protegidas).

\section{Bibliografía}

Allende Landa, J. (2000). Medio ambiente: Ordenación del territorio y sostenibilidad. Bilbao: Universidad del País Vasco.

Asociación Proyecto Sierra De Baza (1998). Guía para conocer y visitar el Parque Natural Sierra de Baza. Granada.

BAigorri, A. (1998). «De la naturaleza social de la Naturaleza». En: PARdo, M. (coord.). Sociología y medio ambiente: Estado de la cuestión. Madrid: Fundación Fernando de los Ríos.

Benton, T.; Redclift, M. (eds.) (1994). Social Theory and the Global Environment. Londres: Routledge. 
COHEN, J.; Rogers, J. (1998). «Solidaridad, democracia y asociación». Zona Abierta, 84-85, p. 177-218.

DOMĖNECH, J. (1999). «El uso y disfrute de los espacios protegidos y de interés: Política forestal y de parques naturales. La protección de la biodiversidad dentro de una estrategia de desarrollo sostenible». En: Grau RaHOLA, J. (coord.). Política ambiental y desarrollo sostenible. Barcelona: Instituto de Ecología y Mercado.

DRYZEK, J. S. (1998). «Ecología y democracia discursiva: más allá del capitalismo liberal y del estado administrativo». Ecología Política, 16, p. 95-109.

EUROPARC-ESPAÑa (1998). Cooperación y resolución de conflictos en espacios naturales protegidos. Comunidad de Madrid.

- (2002). Plan de acción para los espacios naturales protegidos del Estado español. Madrid: Fundación Fernando González Bernáldez..

FuntowicZ, S.; RAVETZ, J. (2000). La ciencia posnormal. Ciencia con la gente. Barcelona: Icaria Antrazyt.

Gibson, C.; Agrawal, A. (2001). Comunities and the environment: etnicity, gender and the state in comunita-based conservation. Rurgers University Press.

Giner, S.; TÁbara, D. (1998). «Piedad cósmica y racionalidad ecológica». Revista Internacional de Sociología, 19 y 20. IESA-CSIC.

Gómez-Limón García, J.; De Lucio Fernández, J. V.; Múgica de la Guerra, M. (2000). De la declaración a la gestión activa: Los espacios naturales protegidos del Estado español en el umbral del siglo XXI. Madrid: Fundación Fernando Gonzáles Bernáldez.

GrASA, R.; Ulied, A. (eds.) (2000). Medio ambiente y gobernabilidad. Barcelona: Icaria. HANNIGAN, J. A. (1991). Environmental sociology: A social constructionist perspective. Londres: Routledge.

Humphrey, M. (2002). Preservation versus the people?: Nature, humanity and political philosophy. Oxford: Oxford University Press.

Mulero Mendigorri, A. (2002). La protección de espacios naturales en España: Antecedentes, contrastes territoriales, conflictos y perspectivas. Madrid: Mundi-Prensa.

RedClift, M.; WoOdgate, G. (coord.) (1997). Sociología del medio ambiente: una perspectiva internacional. Madrid: Mc Graw Hill.

SANZ LOPEZ, C.; SÁNCHEZ Alhama, J. (1998). Medio ambiente y sociedad: de la metáfora organicista a la preservación ecológica. Granada: Comares.

TÁbarA, J. D.; Giner, S. (2004). «Diversity, Civic Virtues and Ecological Austerity». Revue Internationale de Sociologie, vol. 14, no 2, p. 261-285.

TORRES RodrígueZ, A. J. (2003). Estudio sociológico de los espacios naturales protegidos: de la conservación a la sostenibilidad. Universidad de Granada.

WoOdGATE, G.; REDCLIFT, M. (1998). «De una sociología de la naturaleza a una sociología ambiental. Más allá de la construcción social». Revista Internacional de Sociología, 19 y 20. IESA-CSIC.

YounG, I. M. (1998). «Los grupos sociales en la democracia asociativa». Zona Abierta, 84-85, p. 165-175. 